\title{
The Complexity of Set Constraints*
}

\author{
Alexander Aiken ${ }^{1}$, Dexter Kozen ${ }^{2}$, Moshe Vardi $^{3}$, Ed Wimmers ${ }^{4}$ \\ 1 EECS Division, University of California, Berkeley, CA 94720, USA \\ aiken@cs.berkeley . edu \\ 2 Computer Science Department, Cornell University, Ithaca, NY 14853, USA \\ kozenecs. cornell.edu \\ 3 Computer Science Department, Rice University, Houston, TX 77251, USA \\ vardiocs.rice.edu \\ 4 IBM Almaden Research Center, 650 Harry Road, San Jose, CA 95120, USA \\ wimmers@almaden.ibm. com
}

\begin{abstract}
Set constraints are relations between sets of terms. They have been used extensively in various applications in program analysis and type inference. We present several results on the computational complexity of solving systems of set constraints. The systems we study form a natural complexity hierarchy depending on the form of the constraint language.
\end{abstract}

\section{Introduction}

Systems of set constraints have received considerable attention as a formalism for expressing algorithms in program analysis and type inference. Many algorithms based on set constraints have been proposed and implemented, but very little is known about the computational complexity of solving systems of set constraints. In this paper we present complexity results for a natural hierarchy of decision problems involving set constraints.

Set constraints are formal inclusions and negated inclusions between expressions representing subsets of $T_{\Sigma}$, the set of ground terms over a finite ranked alphabet $\Sigma$. A positive set constraint is an inclusion $E \subseteq F$, where $E$ and $F$ are expressions built from a set $X=\{x, y, \ldots\}$ of variables ranging over subsets of $T_{\Sigma}$, a constant 0 denoting the empty set, a constant 1 denoting the set $T_{\Sigma}$, the usual set-theoretic operators $\cup$ (set union), $\cap$ (set intersection), and $\sim$ (complement in $T_{\Sigma}$ ), and an $n$-ary set constructor $f$ for each $n$-ary symbol $f \in \Sigma$ with semantics

$$
f\left(A_{1}, \ldots, A_{n}\right)=\left\{f t_{1} \ldots t_{n} \mid t_{i} \in A_{i}, 1 \leq i \leq n\right\} .
$$

Any valuation $\sigma: X \rightarrow 2^{T_{\Sigma}}$ assigning a subset of $T_{\Sigma}$ to each variable extends uniquely to a map

$$
\sigma:\{\text { set expressions }\} \rightarrow 2^{T_{\Sigma}} .
$$

\footnotetext{
* In E. Börger, Y. Gurevich, and K. Meinke, editors, Proc. 1993 Conf. Computer Science Logic (CSL'93), volume 832 of Lect. Notes in Comput. Sci., pages 1-17. Eur. Assoc. Comput. Sci. Logic, Springer, September 1993.
} 
A valuation $\sigma$ satisfies the constraint $E \subseteq F$ if $\sigma(E) \subseteq \sigma(F)$. A set $\mathcal{S}$ of constraints is satisfiable if there is a valuation that satisfies all constraints in $\mathcal{S}$ simultaneously.

An algorithm for determining the satisfiability of general systems of positive set constraints was first presented in [4]. In this paper, we extend the results of [4] in two ways. In Section 5, we give a new characterization of the satisfiability problem that may be of independent interest. We show that deciding whether $\mathcal{S}$ is satisfiable is equivalent to deciding whether or not a certain finite hypergraph constructed from $\mathcal{S}$ has an induced subhypergraph that is closed (see Section 4). This characterization is simpler than the one in [4] and has the additional advantage for complexity analysis that the hypergraphs can be specified using short Boolean formulas.

In Section 6, we exploit the hypergraph characterization of the satisfiability problem to obtain a family of complexity results for the satisfiability problem. We obtain an exhaustive hierarchy of completeness results for various complexity classes depending on the number of elements of $\Sigma$ of each arity. To the best of our knowledge, these are the first upper and lower bound results for set constraint problems other than the NEXPTIME-completeness result for the general problem, which has been obtained independently in [6]. Our complexity results are summarized in the following table.

\begin{tabular}{|l|l|l|l|}
\hline \multicolumn{3}{|l|}{ number of elements in $\Sigma$ of } & $\begin{array}{l}\text { complexity of the } \\
\text { satisfiability problem }\end{array}$ \\
\hline arity & arity 1 & arity $2+$ & sal \\
\hline \hline 0 & $0+$ & $0+$ & trivial \\
\hline $1+$ & 0 & 0 & NP-complete \\
\hline $1+$ & 1 & 0 & PSPACE-complete \\
\hline $1+$ & $2+$ & 0 & EXPTIME-complete \\
\hline $1+$ & $0+$ & $1+$ & NEXPTIME-complete \\
\hline
\end{tabular}

\section{$2 \quad$ Applications and Related Work}

The greatest interest in set constraints stems from the area of program analysis, where set constraints have been used for a number of years in many different settings $[18,14,16,17,20,13,2,3]$. In these applications, set constraints are generated from the program text and then solved to obtain useful information about the program (e.g., whether it is well-typed). Representing basic data structures such as lists requires binary symbols; our results show that in this general case, solving set constraints is NEXPTIME-complete. In practice, implementations of set constraint solvers introduce restrictions or heuristics specific to the problem domain to achieve better worst-case time complexity. Our results show that such techniques are in fact necessary to achieve more efficient implementations.

Most of the systems for program analysis cited above deal with only positive constraints. In $[2,3]$, opportunities for program optimization are identified by an ad hoc technique for checking the satisfiability of systems of negative set constraints $E \notin F$. The satisfiability of systems of positive and negative constraints 
has been shown to be decidable [1,11], and Stefansson [19] and independently Charatonik and Pacholski [9] have recently shown that the problem is NEXPTIME-complete, thus has the same complexity as positive constraints alone.

Special cases of set constraints have also arisen naturally in the study of finite automata. An example is an algorithm for solving equations between regular languages with free variables [7]. In [7], no complexity analysis is given. There is a simple linear-time reduction of regular expressions to systems of set constraints over unary and nullary symbols. Thus, our results show that deciding the satisfiability of equations between regular languages with free variables is in EXPTIME.

Set constraints with only nullary symbols correspond to Boolean algebras over a finite set of atoms. See [15] for more general results on solving negative constraints in arbitrary Boolean algebras.

Finally, set constraints have been studied for their own sake and several algorithms for solving set constraints have been proposed [12, 4, 10]. Our results differ from these in that we are interested primarily in the complexity of the satisfiability problem for set constraints.

\section{Set Expressions and Set Constraints}

Let $\Sigma$ be a finite ranked alphabet consisting of symbols $f$, each with an associated arity $\operatorname{arity}(f) \in \mathbb{N}$. Symbols in $\Sigma$ of arity $0,1,2,3,4$, and $n$ are called nullary, unary, binary, ternary, quaternary, and $n$-ary, respectively. Nullary elements of $\Sigma$ are often called constants. The set of elements of $\Sigma$ of arity $n$ is denoted $\Sigma_{n}$.

The set of ground terms over $\Sigma$ is denoted $T_{\Sigma}$. This is the smallest set such that if $t_{1}, \ldots, t_{n} \in T_{\Sigma}$ and $f \in \Sigma_{n}$, then $f t_{1} \ldots t_{n} \in T_{\Sigma}$. If $X=\{x, y, \ldots\}$ is a set of variables, then $T_{\Sigma}(X)$ denotes the set of terms over $\Sigma$ and $X$, considering the elements of $X$ as symbols of arity 0 .

Let $\mathrm{B}=(\cup, \cap, \sim, 0,1)$ be the usual signature of set algebra. Let $\Sigma+\mathrm{B}$ denote the signature consisting of the disjoint union of $\Sigma$ and B. A set expression over $X$ is any element of $T_{\Sigma+\mathrm{B}}(X)$. The following is a typical set expression:

$$
f(g(x \cup y), \sim g(x \cap y)) \cup a
$$

where $f \in \Sigma_{2}, g \in \Sigma_{1}, a \in \Sigma_{0}$, and $x, y \in X$. Set expressions are denoted $E, F, \ldots$

A (positive) set constraint is a formal inclusion $E \subseteq F$, where $E$ and $F$ are set expressions. We might also allow equational constraints $E=F$, although inclusions and equations are interdefinable.

We interpret set expressions over the powerset $2^{T_{\Sigma}}$ of $T_{\Sigma}$. This forms an algebra of signature $\Sigma+\mathrm{B}$ where the Boolean operators have their usual settheoretic interpretations and elements $f \in \Sigma_{n}$ are interpreted as set functions

$$
f\left(A_{1}, \ldots, A_{n}\right)=\left\{f t_{1} \ldots t_{n} \mid t_{i} \in A_{i}, 1 \leq i \leq n\right\} .
$$

A set assignment is a map $\sigma: X \rightarrow 2^{T_{\Sigma}}$ assigning a subset of $T_{\Sigma}$ to each variable in $X$. Any set assignment $\sigma$ extends uniquely to a ( $\Sigma+\mathrm{B}$ )-homomorphism

$$
\sigma: T_{\Sigma+\mathrm{B}}(X) \rightarrow 2^{T_{\Sigma}}
$$


by induction on the structure of the set expression in the usual way. The set assignment $\sigma$ satisfies the constraint $E \subseteq F$ if $\sigma(E) \subseteq \sigma(F)$. A family $\mathcal{S}$ of set constraints is satisfiable if there is a set assignment that satisfies all the constraints in $\mathcal{S}$ simultaneously. The satisfiability problem is to determine whether a given finite system $\mathcal{S}$ of set constraints over $\Sigma$ is satisfiable.

A Boolean expression over $X$ is any element of $T_{\mathrm{B}}(X)$ (i.e., a set expression with no symbols from $\Sigma$ ). A truth assignment is a map $u: X \rightarrow \mathbf{2}$ where $\mathbf{2}=\{0,1\}$ is the two-element Boolean algebra. Any truth assignment $u$ extends uniquely to a B-homomorphism $u: T_{\mathrm{B}}(X) \rightarrow \mathbf{2}$ inductively according to the rules of Boolean algebra. If $X=\left\{x_{1}, \ldots, x_{m}\right\}$, we use the notation $B\left[x_{i}:=a_{i}\right]$ to denote the truth value of the Boolean formula $B$ under the truth assignment $x_{i} \mapsto a_{i}, 1 \leq i \leq m$.

\subsection{Normal Form}

We show in this section how to transform a given system $\mathcal{S}$ of set constraints into an equivalent system in a special normal form. This step simplifies the proof of correspondence between set constraints and hypergraphs, because the normal form is actually quite close to the hypergraphs defined in Section 4. The transformation is linear for fixed $\Sigma$.

1. For every subexpression $f E_{1} \ldots E_{n}$ of some set expression in $\mathcal{S}$, where $f \in \Sigma_{n}$, let $y_{0}, y_{1}, \ldots, y_{n}$ be new variables, replace the subexpression $f E_{1} \ldots E_{n}$ by $y_{0}$, and add new constraints $y_{0}=f y_{1} \ldots y_{n}$ and $y_{i}=E_{i}, 1 \leq i \leq n$. Continue until all constraints are either purely Boolean constraints (i.e., do not contain any occurrence of $f \in \Sigma$ ) or are of the form $y_{0}=f y_{1} \ldots y_{n}$ where $f \in \Sigma_{n}$ and $y_{0}, y_{1}, \ldots, y_{n}$ are variables. Let $X=\left\{x_{1}, \ldots, x_{m}\right\}$ be the set of all variables occurring in $\mathcal{S}$ at this point.

2. For each $f \in \Sigma_{n}$, introduce a new set of variables

$$
Z_{f}=\left\{z_{i j}^{f} \mid 0 \leq i \leq n, 1 \leq j \leq m\right\}
$$

and add the constraints

$$
z_{0 j}^{f}=f \underbrace{1 \ldots 1}_{n} \cap x_{j} \quad z_{i j}^{f}=f \underbrace{1 \ldots 1}_{i-1} x_{j} \underbrace{1 \ldots 1}_{n-i}
$$

for all $1 \leq i \leq n$ and $1 \leq j \leq m$.

3. Each constraint $x_{j_{0}}=f x_{j_{1}} \ldots x_{j_{n}}$ obtained in step 1 is equivalent to the constraint

$$
x_{j_{0}}=f x_{j_{1}} \underbrace{1 \ldots 1}_{n-1} \cap f 1 x_{j_{2}} \underbrace{1 \ldots 1}_{n-2} \cap \cdots \cap f \underbrace{1 \ldots 1}_{n-1} x_{j_{n}}
$$

which in turn is equivalent to the conjunction of constraints

$$
\begin{aligned}
& f \underbrace{1 \ldots 1}_{n} \cap x_{j_{0}}=f x_{j_{1}} \underbrace{1 \ldots 1}_{n-1} \cap f 1 x_{j_{2}} \underbrace{1 \ldots 1}_{n-2} \cap \cdots \cap f \underbrace{1 \ldots 1}_{n-1} x_{j_{n}} \\
& g \underbrace{1 \ldots 1}_{m} \cap x_{j_{0}}=0, g \neq f, m=\operatorname{arity}(g) .
\end{aligned}
$$


Replace the constraint $x_{j_{0}}=f x_{j_{1}} \ldots x_{j_{n}}$ with the constraints

$$
z_{0 j_{0}}^{f}=\bigcap_{i=1}^{n} z_{i j_{i}}^{f} \quad z_{0 j_{0}}^{g}=0, g \neq f .
$$

Because of the constraints introduced in step 2, the resulting system is equivalent.

4. At this point we have

- purely Boolean constraints formed in step 1 involving only the variables $X$

- for each $f \in \Sigma$, purely Boolean constraints formed in step 3 involving only the variables $Z_{f}$

- the mixed constraints formed in step 2.

Replace each purely Boolean constraint $E \subseteq F$ involving the variables $X$ by the equivalent constraint $\sim E \cup F=1$. Let $B$ be the conjunction of all the left hand sides of such constraints, and replace all these constraints in $\mathcal{S}$ with the single constraint $B=1$. Do the same for the purely Boolean constraints involving the variables $Z_{f}$ to get a single constraint $C_{f}=1$ for each $f \in \Sigma$.

After this translation, $\mathcal{S}$ contains

- a constraint $B=1$, where $B \in T_{\mathrm{B}}(X)$

- for each $f \in \Sigma$, a constraint $C_{f}=1$, where $C_{f} \in T_{\mathrm{B}}\left(Z_{f}\right)$

- constraints

$$
z_{0 j}^{f}=f \underbrace{1 \ldots 1}_{n} \cap x_{j} \quad z_{i j}^{f}=f \underbrace{1 \ldots 1}_{i-1} x_{j} \underbrace{1 \ldots 1}_{n-i}
$$

for each $f \in \Sigma_{n}$ and each $1 \leq i \leq n, 1 \leq j \leq m$.

This is the desired normal form.

\section{Hypergraphs}

For our purposes, a hypergraph is a structure $H=\left(U, E_{i} \mid i \in I\right)$ consisting of a finite set $U$ of vertices and an indexed family of relations $E_{i}$ of various arities on $U$ called hyperedge relations. An element of an $n$-ary hyperedge relation is called an $n$-ary hyperedge. In our application, the index set $I$ is the ranked alphabet $\Sigma$, and for $f \in \Sigma$, $\operatorname{arity}\left(E_{f}\right)$ is $\operatorname{arity}(f)+1$.

If $U^{\prime} \subseteq U$, the induced subhypergraph of $H$ on $U^{\prime}$ is the hypergraph $H^{\prime}$ on vertices $U^{\prime}$ whose hyperedge relations are the hyperedge relations of $H$ restricted to $U^{\prime}$. That is, $H^{\prime}=\left(U^{\prime}, E_{i}^{\prime} \mid i \in I\right)$ where if $E_{i}$ is $n$-ary then $E_{i}^{\prime}=E_{i} \cap\left(U^{\prime}\right)^{n}$.

An $(n+1)$-ary hyperedge relation $E$ of the hypergraph $H$ is closed if for each $n$-tuple $u_{1}, \ldots, u_{n} \in U^{n}$, there exists $u_{0} \in U$ such that $\left(u_{0}, u_{1}, \ldots, u_{n}\right) \in E$. In the case $n=0$, this definition just says $E \cap U \neq \emptyset$. Abusing notation, we can think of $E$ as a function $E: U^{n} \rightarrow 2^{U}$ where

$$
E\left(u_{1}, \ldots, u_{n}\right)=\left\{u_{0} \mid\left(u_{0}, u_{1}, \ldots, u_{n}\right) \in E\right\} .
$$


In this view the hyperedge relation $E$ is closed iff $E\left(u_{1}, \ldots, u_{n}\right) \neq \emptyset$ for each $n$ tuple $u_{1}, \ldots, u_{n} \in U^{n}$. The hypergraph $H$ is closed if all its hyperedge relations are closed.

The hypergraph closure problem is the problem of determining whether a given hypergraph has a closed induced subhypergraph.

Example 1. Consider the hypergraph consisting of vertices $\mathbb{Z}_{p}$, the field of integers modulo a prime $p$, and a single ternary hyperedge relation

$$
E=\left\{(a, b, a b) \mid a, b \in \mathbb{Z}_{p}\right\} .
$$

This hypergraph is not closed because there is no $a \in \mathbb{Z}_{p}$ such that $(a, 0,1) \in E$. However, the induced subhypergraph on the nonzero elements of $\mathbb{Z}_{p}$ is closed, since for all $b, c \in \mathbb{Z}_{p}-\{0\}$ there exists an $a \in \mathbb{Z}_{p}-\{0\}$ such that $(a, b, c) \in E$.

\subsection{Succinct Specification of Hypergraphs}

We work with a particular class of hypergraphs whose vertices and hyperedge relations are specified succinctly by Boolean formulas in the following way. Let

$$
X=\left\{x_{1}, \ldots, x_{m}\right\} \quad Z_{f}=\left\{z_{i j}^{f} \mid 0 \leq i \leq \operatorname{arity}(f), 1 \leq j \leq m\right\}, f \in \Sigma
$$

be pairwise disjoint sets of variables. Suppose we are given Boolean formulas

$$
B \in T_{\mathrm{B}}(X) \quad C_{f} \in T_{\mathrm{B}}\left(Z_{f}\right), f \in \Sigma .
$$

These formulas determine a hypergraph $H=\left(U, E_{f} \mid f \in \Sigma\right)$ as follows. The vertex set $U$ is the set of all truth assignments $u: X \rightarrow \mathbf{2}$ satisfying $B$. Each such truth assignment corresponds to a conjunction of literals (also denoted $u$ ) in which each variable in $X$ occurs exactly once, either positively or negatively, such that $u \subseteq B$ tautologically. The variable $x$ occurs positively iff $u(x)=1$. We occasionally call the elements of $U$ atoms, because they represent atoms of the free Boolean algebra on generators $X$ modulo $B=1$. Each Boolean expression over $X$ is equivalent modulo $B=1$ to a disjunction of atoms.

For each $f \in \Sigma_{n}$, the hyperedge relation $E_{f}$ of $H$ is defined to be the set of all $(n+1)$-tuples $\left(u_{0}, \ldots, u_{n}\right) \in U^{n+1}$ such that

$$
C_{f}\left[z_{i j}^{f}:=u_{i}\left(x_{j}\right)\right]=1 .
$$

Intuitively, we think of the formula $C_{f}$ as a Boolean valued mapping on $(n+1)$ tuples of truth assignments to $X$. To emphasize this intuition, we abbreviate the left hand side of (4) by $C_{f}\left[u_{0}, \ldots, u_{n}\right]$. Thus

$$
\left(u_{0}, \ldots, u_{n}\right) \in E_{f} \text { iff } C_{f}\left[u_{0}, \ldots, u_{n}\right]=1 .
$$

In general, the size of the hypergraph can be exponential in the size of its specification. 


\section{Set Constraints and Hypergraph Closure}

In this section we give an efficiently computable correspondence between systems of set constraints in normal form as described in Section 3.1 and hypergraphs specified by systems of Boolean formulas as described in Section 4.1. Let $X$ and $Z_{f}$ be sets of variables as described in (2). The system $\mathcal{S}$ in normal form consisting of constraints $B$ and $C_{f}, f \in \Sigma$, as described in (3), along with the constraints (1), corresponds to the hypergraph $H$ specified by the formulas $B$ and $C_{f}, f \in \Sigma$.

Theorem 1. The hypergraph $H$ has a closed induced subhypergraph if and only if the system $\mathcal{S}$ of set constraints is satisfiable.

Proof. $(\Rightarrow)$ Let $H^{\prime}=\left(U^{\prime}, E_{f}^{\prime} \mid f \in \Sigma\right)$ be a closed induced subhypergraph of $H$. Define $\theta: T_{\Sigma} \rightarrow U^{\prime}$ inductively such that for all $f \in \Sigma_{n}$ and $t_{1}, \ldots, t_{n} \in T_{\Sigma}$,

$$
\theta\left(f t_{1} \ldots t_{n}\right) \in E_{f}^{\prime}\left(\theta\left(t_{1}\right), \ldots, \theta\left(t_{n}\right)\right) .
$$

This is possible since $H^{\prime}$ is closed. Each $\theta(t)$ is a truth assignment to $X$ satisfying $B$. For each term $t_{0}$ of the form $f t_{1} \ldots t_{n}$, extend $\theta\left(t_{0}\right)$ to a truth assignment to $X \cup Z_{f}$ as follows:

$$
\theta\left(t_{0}\right)\left(z_{i j}^{f}\right)=\theta\left(t_{i}\right)\left(x_{j}\right), z_{i j}^{f} \in Z_{f} .
$$

Define a set assignment $\sigma$ by

$$
\begin{aligned}
\sigma\left(x_{j}\right) & =\left\{t \mid \theta(t)\left(x_{j}\right)=1\right\} \\
\sigma\left(z_{i j}^{f}\right) & =\sigma(f \underbrace{1 \ldots 1}_{i-1} x_{j} \underbrace{1 \ldots 1}_{n-i}) \\
\sigma\left(z_{0 j}^{f}\right) & =\sigma(f \underbrace{1 \ldots 1}_{n} \cap x_{j})
\end{aligned}
$$

for $f \in \Sigma_{n}, 1 \leq i \leq n$, and $1 \leq j \leq m$. We show that $\sigma$ satisfies $\mathcal{S}$.

It is immediate from the definition of $\sigma$ that the constraints (1) are satisfied. We now show that $\sigma$ satisfies $B=1$. For $t \in T_{\Sigma}$, let $\chi_{t}: 2^{T_{\Sigma}} \rightarrow \mathbf{2}$ be the characteristic function

$$
\chi_{t}(A)= \begin{cases}1, & \text { if } t \in A, \\ 0, & \text { if } t \notin A .\end{cases}
$$

By definition of $\sigma$,

$$
\chi_{t}\left(\sigma\left(x_{j}\right)\right)=1 \Leftrightarrow t \in \sigma\left(x_{j}\right) \Leftrightarrow \theta(t)\left(x_{j}\right)=1
$$

so $\chi_{t} \circ \sigma$ and $\theta(t)$ agree on $X$. Since both are B-homomorphisms (i.e., preserve Boolean operations), they agree on all elements of $T_{\mathrm{B}}(X)$; in particular, they agree on $B$. Since $\theta(t)(B)=1$ for all $t$ by definition of $U$, we have that $\chi_{t}(\sigma(B))=1$ for all $t$. Thus $\sigma(B)=T_{\Sigma}=\sigma(1)$.

Finally, we show that $\sigma$ satisfies $C_{f}=1$. Recall that $C_{f}$ is a conjunction of set expressions of the form $\sim E \cup F$, where $E$ and $F$ are either 0 or a conjunction 
of elements of $Z_{f}$. Since $\sigma$ satisfies (1), it also satisfies $E \subseteq f 1 \ldots 1$. By Boolean reasoning it follows that $\sigma$ also satisfies $\sim f 1 \ldots 1 \subseteq \sim E \subseteq \sim E \cup F$, therefore $\sim f 1 \ldots 1 \subseteq C_{f}$. Thus it remains to show that $\sigma$ satisfies $f 1 \ldots 1 \subseteq C_{f}$, or in other words, $t_{0} \in \sigma\left(C_{f}\right)$ for all terms $t_{0}$ of the form $f t_{1} \ldots t_{n}$. As above, we argue that $\chi_{t_{0}} \circ \sigma$ and $\theta\left(t_{0}\right)$ agree on $Z_{f}$ :

$$
\begin{aligned}
\theta\left(t_{0}\right)\left(z_{i j}^{f}\right) & =\theta\left(t_{i}\right)\left(x_{j}\right) & & \text { by }(5) \\
& =t_{i} \in \sigma\left(x_{j}\right) & & \text { by }(6) \\
& =t_{0} \in \sigma(f \underbrace{1 \ldots 1}_{i-1} x_{j} \underbrace{1 \ldots 1}_{n-i}) & & \text { since } \sigma \text { is a homomorphism } \\
& =\chi_{t_{0}}\left(\sigma\left(z_{i j}^{f}\right)\right) & & \text { by definition of } \sigma
\end{aligned}
$$

for $1 \leq i \leq n$ and $1 \leq j \leq m$. Similar reasoning applies to $z_{0 j}^{f}$. Since both $\chi_{t_{0}} \circ \sigma$ and $\theta\left(t_{0}\right)$ are B-homomorphisms, they agree on all elements of $T_{\mathrm{B}}\left(Z_{f}\right)$; in particular, they agree on $C_{f}$. Since $\left(\theta\left(t_{0}\right), \theta\left(t_{1}\right), \ldots, \theta\left(t_{n}\right)\right) \in E_{f}^{\prime}$, we have

$$
\begin{aligned}
C_{f}\left[\theta\left(t_{0}\right), \ldots, \theta\left(t_{n}\right)\right]=1 & \Rightarrow C_{f}\left[z_{i j}^{f}:=\theta\left(t_{i}\right)\left(x_{j}\right)\right]=1 \\
& \Rightarrow C_{f}\left[z_{i j}^{f}:=\theta\left(t_{0}\right)\left(z_{i j}^{f}\right)\right]=1 \\
& \Rightarrow \theta\left(t_{0}\right)\left(C_{f}\right)=1 \\
& \Rightarrow \chi_{t_{0}}\left(\sigma\left(C_{f}\right)\right)=1 \\
& \Rightarrow t_{0} \in \sigma\left(C_{f}\right) .
\end{aligned}
$$

$(\Leftarrow)$ Suppose $\sigma$ satisfies $\mathcal{S}$. Regarding $u \in U$ as a conjunction of literals over $X$, let $U^{\prime}=\{u \mid \sigma(u) \neq \emptyset\}$. Since $\sigma$ satisfies $\mathcal{S}$, it satisfies $B$, therefore $U^{\prime} \subseteq U$. We claim that the induced subhypergraph $H^{\prime}=\left(U^{\prime}, E_{f}^{\prime} \mid f \in \Sigma\right)$ of $H$ is closed. For $f \in \Sigma_{n}$ and for all $u_{1}, \ldots, u_{n} \in U^{\prime}$, let $t_{i} \in \sigma\left(u_{i}\right), 1 \leq i \leq n$. The $t_{i}$ exist by definition of $U^{\prime}$. There is a unique atom $u_{0}$ such that $t_{0}=f t_{1} \ldots t_{n} \in \sigma\left(u_{0}\right)$, and $u_{0} \in U^{\prime}$.

Extend $u_{0}$ to domain $X \cup Z_{f}$ by defining $u_{0}\left(z_{i j}^{f}\right)=u_{i}\left(x_{j}\right), 0 \leq i \leq n, 1 \leq j \leq$ $m$. Then

$$
\begin{aligned}
u_{0}\left(z_{i j}^{f}\right) & =u_{i}\left(x_{j}\right) \\
& =\left\{\begin{array}{l}
1, \text { if } t_{i} \in \sigma\left(x_{j}\right) \\
0, \text { otherwise }
\end{array}\right. \\
& =\left\{\begin{array}{l}
1, \text { if } i=0 \text { and } t_{0} \in \sigma\left(x_{j}\right) \\
1, \text { if } i \geq 1 \text { and } t_{0} \in \sigma(f \underbrace{1 \ldots 1}_{i-1} x_{j} \underbrace{1 \ldots 1}_{n-i}) \\
0, \text { otherwise }
\end{array}\right. \\
& =\left\{\begin{array}{l}
1, \text { if } i=0 \text { and } t_{0} \in \sigma\left(z_{0 j}^{f}\right) \\
1, \text { if } i \geq 1 \text { and } t_{0} \in \sigma\left(z_{i j}^{f}\right) \\
0, \text { otherwise }
\end{array}\right. \\
& =\chi_{t_{0}}\left(\sigma\left(z_{i j}^{f}\right)\right) .
\end{aligned}
$$


Since $u_{0}$ and $\chi_{t_{0}} \circ \sigma$ agree on $Z_{f}$ and both are B-homomorphisms, they agree on $C_{f}$. Thus $u_{0}\left(C_{f}\right)=\chi_{t_{0}}\left(\sigma\left(C_{f}\right)\right)=1$, since $\sigma$ satisfies $C_{f}=1$. Then

$$
\begin{aligned}
u_{0}\left(C_{f}\right)=1 & \Rightarrow C_{f}\left[z_{i j}^{f}:=u_{0}\left(z_{i j}^{f}\right)\right]=1 \\
& \Rightarrow C_{f}\left[z_{i j}^{f}:=u_{i}\left(x_{j}\right)\right]=1 \\
& \Rightarrow C_{f}\left[u_{0}, u_{1}, \ldots, u_{n}\right]=1 \\
& \Rightarrow\left(u_{0}, u_{1}, \ldots, u_{n}\right) \in E_{f} .
\end{aligned}
$$

Since the $u_{i}$ are in $U^{\prime}$, we also have $\left(u_{0}, u_{1}, \ldots, u_{n}\right) \in E_{f}^{\prime}$. Thus $H^{\prime}$ is closed.

Corollary 2. The following two decision problems are linearly interreducible:

(i) Given a system $\mathcal{S}$ of set constraints, is it satisfiable?

(ii) Given a hypergraph $H$ specified by Boolean formulas, does it have a closed induced subhypergraph?

\section{Complexity Bounds}

In this section we give complexity bounds for a hierarchy of satisfiability problems for systems of set constraints based on the arities of the elements of $\Sigma$. By Theorem 1, we are free to work with either the constraints $\mathcal{S}$ directly or the hypergraph $H$. It is usually easier to deal with $H$ because it is a finite object, whereas in general $\mathcal{S}$ involves infinitely many terms and can have infinitely many solutions.

The results of this section are summarized in the table in Section 1. The first line of the table is really a triviality, because with no constants in $\Sigma$, the set of ground terms $T_{\Sigma}$ is empty. We handle each of the other cases separately.

\subsection{Nullary Symbols}

With at least one constant in $\Sigma$ but no symbol of higher arity, we have $T_{\Sigma}=\Sigma=$ $\Sigma_{0}$. In this case the satisfiability problem is $N P$-complete. The hypergraph $H$ has only unary hyperedge relations, and the closure problem amounts to determining whether for each $c \in \Sigma$ there exists a truth assignment $u$ satisfying both $B$ and $C_{c}$ (in the sense that $u(B)=1$ and $C_{c}[u]=1$ ). This is essentially Boolean satisfiability.

\subsection{One Unary Symbol}

With one unary symbol, the problem is PSPACE-complete. For the upper bound, suppose $\Sigma$ consists of one unary symbol $f$, one or more constants, and no other symbols. Then the hypergraph $H$ is simply a conventional directed graph with binary edge relation $E_{f}$ and a distinguished subset $E_{c}$ for each constant $c$. The vertices $U$ are the truth assignments satisfying a Boolean formula $B$, the edge relation $E_{f}$ is the set of pairs $(u, v) \in U^{2}$ such that $C_{f}[u, v]=1$, and the distinguished subset $E_{c}$ of $U$ is the set of $u$ such that $C_{c}[u]=1$. In this case, the closure problem is to determine whether there is a subset $U^{\prime}$ of $U$ such that 
- each $E_{c}$ intersects $U^{\prime}$

- for any $v \in U^{\prime}$ there is a $u \in U^{\prime}$ such that $(u, v) \in E_{f}$.

This can be determined nondeterministically in linear space as follows. For each constant $c$ in turn, guess $u \in E_{c}$ and verify that $E_{c}[u]=u(B)=1$. Starting from $u$, guess an $E_{f}$-path of length $2^{m}+1$, where $m=|X|$. At each step, verify the new vertex $v$ by evaluating $v(B)$ and the new edge $(v, w)$ by evaluating $C_{f}[v, w]$. If such a path is found for all $c$, accept.

Since $|U| \leq 2^{m}$, any $E_{f}$-path of length $2^{m}+1$ must repeat a vertex. Thus the procedure accepts iff for every nullary $c \in \Sigma$ there is an $E_{f}$-path $u_{0}, u_{1}, \ldots, u_{k}$ such that $u_{0} \in E_{c}$ and $u_{k}=u_{j}$ for some $j<k$. The induced hypergraph on the set of all such $u_{i}$ for all $c$ is closed. Conversely, any closed induced subhypergraph must contain an induced subhypergraph of this form.

This procedure requires only linear space to record the current vertex, $m$ bits for a counter, and enough space to evaluate the formulas. Thus the algorithm runs in nondeterministic PSPACE, which is the same as deterministic PSPACE by Savitch's Theorem.

We show that the problem of deciding whether a system with one unary symbol $f$ and one constant $c$ is satisfiable is PSPACE-hard by a reduction from the halting problem for linear-bounded automata (LBA), a well known PSPACEcomplete problem. Consider an LBA $M$ and input string $w$ of length $n$. A configuration of $M$ on input $w$ is an instantaneous description of $M$ 's current tape contents, state, and head position. Each legal configuration is represented as a string of symbols of length $n$ over a finite alphabet. Assume without loss of generality that there is a unique accept configuration and a unique reject configuration on inputs of length $n$, that all computation paths of $M$ halt and either accept or reject (equip $M$ with a binary exponential-time counter if necessary), that the accept configuration enters a trivial loop, and that the reject configuration has no successor.

The configurations of $M$ on $w$ can be encoded as truth assignments to Boolean variables

$$
\begin{aligned}
& A_{j}^{a}=\text { "symbol } a \text { is written on tape cell } j ", 1 \leq j \leq n, a \in \Gamma \\
& Q_{j}^{q}=\text { " } M \text { is in state } q \text { scanning tape cell } j ", 1 \leq j \leq n, q \in Q .
\end{aligned}
$$

These variables comprise the set $X$. One can write down short formulas describing the action of $M$ on input $w$ :

- a formula $B$ describing all legal configurations (exactly one symbol occupying each tape cell, exactly one current state, exactly one tape cell currently being scanned);

- a formula $C_{c}$ describing the start configuration of $M$ on input $w$ (the symbol occupying tape cell $j$ is the $j^{\text {th }}$ symbol of $w$ and $M$ is in state $s$ scanning the leftmost tape cell);

- a formula $C_{f}[u, v]$ describing legal pairs of configurations such that $u$ follows from $v$ in one step according to the transition rules of $M$. 
The encoding technique is similar to that used in the proof of Cook's Theorem. Then $M$ accepts input $w$ if and only if the hypergraph specified by $B, C_{c}$, and $C_{f}$ has a closed induced subhypergraph consisting of the configurations in the accepting computation path.

\subsection{Two or More Unary Symbols}

With two or more unary symbols, one or more constant symbols, and no other symbols, consider the following deterministic exponential-time algorithm for determining whether the specified hypergraph has a closed induced subhypergraph. Write down all truth assignments to $X$ and delete those not satisfying $B$. For each remaining $u$, check whether it has an $E_{f}$-successor for all unary $f$ and delete it if not (inductively, such a $u$ cannot be contained in any closed subhypergraph). Repeat until no more vertices are deleted. The procedure succeeds if not all vertices are deleted and for each nullary $c$ there is a $u \in E_{c}$. In that case, the resulting subhypergraph contains all closed subhypergraphs, and is closed itself. There are at most $2^{m}$ truth assignments, and the tests can be done efficiently by evaluating $C_{f}[u, v]$ and $C_{e}[u]$.

The exponential time hardness for two unary symbols is obtained by generalizing the lower bound construction for one unary symbol. Instead of a deterministic LBA, we encode an alternating LBA $M$ on input $w$ [8]. We assume without loss of generality that $M$ has no negating transitions, that there is a unique accept and a unique reject configuration for inputs of length $n$, that $M$ alternates strictly between universal and existential branches, that all branches are at most binary, that the start, accept, and reject configurations are universal branches, that all computation paths either accept or reject, that the unique accept configuration enters a trivial loop, and that the unique reject configuration has no successor. We will construct a hypergraph that has a closed induced subhypergraph iff $M$ accepts $w$.

Let $\alpha$ be a universal configuration with successors $\alpha 0$ and $\alpha 1$ in lexicographical order. By assumption, $\alpha 0$ and $\alpha 1$ are existential configurations. Let $\alpha 00$ and $\alpha 01$ be the two successors of $\alpha 0$ and let $\alpha 10$ and $\alpha 11$ be the two successors of $\alpha 1$ in lexicographical order. Then $\alpha 00, \alpha 01, \alpha 10$, and $\alpha 11$ are universal configurations.

As in Section 6.2, we let $B$ and $C_{e}$ be Boolean formulas describing the set of all legal configurations and the start configuration, respectively. In addition, we let $C_{f}$ describe the relation consisting of all pairs $(\alpha, \alpha 00)$ and $(\alpha, \alpha 01)$, and we let $C_{g}$ describe the relation consisting of all pairs $(\alpha, \alpha 10)$ and $(\alpha, \alpha 11)$. The idea is that in the semantics of alternating Turing machines, $\alpha$ leads to acceptance iff both $\alpha 0$ and $\alpha 1$ lead to acceptance, which occurs iff at least one of $\alpha 00$ or $\alpha 01$ leads to acceptance and at least one of $\alpha 10$ or $\alpha 11$ lead to acceptance. Thus $M$ accepts the input $w$ iff there is a closed induced subhypergraph consisting of an accepting computation tree of $M$. 


\subsection{One or More Binary Symbols}

With any number of symbols of any arity, we can determine in nondeterministic exponential time whether there exists a closed induced subhypergraph by guessing a subset $U^{\prime} \subseteq U$ and verifying that the induced subhypergraph on $U^{\prime}$ is closed; i.e., for all $u \in U^{\prime}, u(B)=1$ and for all $f \in \Sigma_{n}$ and $u_{1}, \ldots, u_{n} \in U^{\prime}$, there exists a $u_{0} \in E_{f}\left(u_{1}, \ldots, u_{n}\right) \cap U^{\prime}$. The set $U$ has at most $2^{m}$ elements, where $m$ is the number of variables, and the predicates $C_{f}\left[u_{0}, \ldots, u_{n}\right]$ require polynomial time to evaluate. The entire algorithm runs in nondeterministic exponential time.

To show that the problem is hard for NEXPTIME, we show that with a constant $c$ and one ternary symbol $f$, we can encode computations of a nondeterministic exponential-time Turing machine. In Section 6.5 below we show how to reduce $f$ to binary. Let $M$ be such a machine with time and space bound $N=2^{O(n)}$. Without loss of generality, assume that $M$ starts in its start state scanning the left endmarker $\vdash$, that it has unique accept and reject configurations on inputs of length $n$ and that all computation paths lead to acceptance or rejection, that all nondeterministic branches are at most binary, that once $M$ accepts or rejects it enters a trivial loop in which it remains in the same state.

Computation histories of $M$ on inputs of length $n$ can be represented as $N \times N$ matrices. Each row $i$ of the matrix encodes a possible configuration of $M$ at time $i$. The $i j^{\text {th }}$ entry of the matrix records the symbol occupying the $j^{\text {th }}$ tape cell at time $i$ and whether that cell is being scanned by the machine at time $i$. If so, the current state of the finite control is also recorded. An accepting computation history of $M$ on input $w$ is represented by a matrix whose first row encodes the start configuration of $M$ on input $w$, whose $i+1^{\text {st }}$ row follows from the $i^{\text {th }}$ by the transition rules of $M$, and whose final row encodes the accept configuration.

Given $M$ and input $w=w_{1} \ldots w_{n}$, we construct Boolean formulas $B, C_{c}$ and $C_{f}$ specifying a hypergraph $H=\left(U, E_{c}, E_{f}\right)$ where $c$ is nullary and $f$ is ternary (thus $E_{c}$ is a unary and $E_{f}$ is quaternary). Each entry of the matrix is represented by a vertex of the hypergraph, which is a truth assignment satisfying $B$. The hyperedge relation $E_{f}$ enforces constraints between adjacent entries. The hypergraph has a closed induced subhypergraph if and only if there exists a matrix representing an accepting computation history of $M$.

We first define the set of Boolean variables $X$. There is a variable $A_{a}$ for each symbol $a$ of the tape alphabet, a variable $Q_{q}$ for each machine state $q, m=$ $\left\lceil\log _{2} N\right\rceil$ variables $t_{0}, \ldots, t_{m-1}$ encoding the time (row number of the matrix) in binary, $m$ variables $s_{0}, \ldots, s_{m-1}$ encoding the position of the tape cell (column of the matrix) in binary, and a variable choice determining the nondeterministic choice.

The vertices of the hypergraph are the truth assignments to $X$ such that at most one state and exactly one tape symbol have Boolean value 1 . This is specified by the formula

$$
B=\left(\bigcup_{a} A_{a}\right) \cap \bigcap_{a \neq b}\left(\sim A_{a} \cup \sim A_{b}\right) \cap \bigcap_{p \neq q}\left(\sim Q_{p} \cup \sim Q_{q}\right)
$$

Each truth assignment $u$ to $X$ corresponds to an index $i j$ into the array, where 
$i, j$ are the numbers $0 \leq i, j \leq N-1$ encoded in binary by the truth values $u\left(t_{k}\right)$ and $u\left(s_{k}\right), 0 \leq k \leq m-1$. We denote $i$ and $j$ by time $(u)$ and space $(u)$, respectively. Also, each $u$ satisfying $B$ has exactly one symbol $a$ with $u\left(A_{a}\right)=1$, which we denote by $\operatorname{sym}(u)$. Finally, each $u$ satisfying $B$ has at most one state $q$ with $u\left(Q_{q}\right)=1$, which we denote by state $(u)$. If $u\left(Q_{q}\right)=0$ for all states $q$, we write state $(u)=\perp$.

We will need to perform modular addition and comparisons on numbers in the range $0 \leq i \leq N-1$ in terms of their binary representations. For example, we need formulas expressing conditions such as time $(u)=\operatorname{time}(v)+1$ and time $(u)>$ time $(v)$. These constructions are quite easy and well known, so we omit the details.

The unary predicate $C_{c}$ specifies the $00^{\text {th }}$ entry (upper left corner) of the matrix. It specifies that the machine is in its start state $s$ scanning the leftmost tape cell, which contains the left endmarker $\vdash$ :

$$
C_{e}[u]=(\operatorname{time}(u)=0) \cap(\operatorname{space}(u)=0) \cap Q_{s} \cap A_{\vdash} .
$$

There are exactly two truth assignments satisfying $B$ and $C_{c}$, one for each value of choice, and one of these must be contained in any closed subhypergraph.

The quaternary predicate $C_{f}[u, v, w, x]$ serves several purposes. It is defined as the conjunction of several formulas describing the format of configurations, the initial configuration (first row of the matrix), the final configuration (last row of the matrix), and the legal transitions.

First we specify that there is at most one truth assignment for every $i j$ :

$$
(\operatorname{time}(w)=\operatorname{time}(x) \cap \operatorname{space}(w)=\operatorname{space}(x)) \Rightarrow w=x .
$$

Inclusion of this formula as a conjunct of $C_{f}[u, v, w, x]$ guarantees that there can be no closed induced subhypergraph containing two distinct vertices $w$ and $x$ such that time $(w)=\operatorname{time}(x)$ and $\operatorname{space}(w)=\operatorname{space}(x)$.

We also wish to specify that for every $i$, the value of the variable choice at all tape cells in row $i$ of the matrix is the same:

$$
\operatorname{time}(w)=\operatorname{time}(x) \Rightarrow(w(\text { choice })=x(\text { choice })) .
$$

To specify the initial configuration, we must ensure that the first $n$ tape cells after the left endmarker contain the input string $w=w_{1} \cdots w_{n}$, that all remaining cells to their right except the last contain the blank symbol $t$, the last tape cell contains the right endmarker $\dashv$, and no other cell besides the leftmost contains a state:

$$
\begin{aligned}
& (v=w=x \cap \operatorname{time}(v)=0 \cap \operatorname{space}(v)<N-1) \\
& \Rightarrow(\text { time }(u)=0 \cap \operatorname{space}(u)=\operatorname{space}(v)+1 \cap \operatorname{state}(u)=\perp \\
& \cap \bigcap_{i=1}^{n}\left(\operatorname{space}(u)=i \Rightarrow \operatorname{sym}(u)=w_{i}\right) \\
& \cap n<\operatorname{space}(u)<N-1 \Rightarrow \operatorname{sym}(u)=\downarrow \\
& \cap \operatorname{space}(u)=N-1 \Rightarrow \operatorname{sym}(u)=-1) .
\end{aligned}
$$


If the premise of (8) is true of $v, w$ and $x$, then there is exactly one choice of $u$ that satisfies the conclusion. The two truth assignments satisfying $C_{c}$ satisfy the premise of (8), and it can be shown inductively that any closed subhypergraph must contain the entire first row of the matrix representing the initial configuration.

To capture valid transitions of the machine, we write

$$
\begin{aligned}
& (\operatorname{time}(v)=\operatorname{time}(w)=\operatorname{time}(x)<N-1 \\
& \cap \operatorname{space}(x)=\operatorname{space}(w)+1=\operatorname{space}(v)+2) \\
& \Rightarrow(\operatorname{time}(u)=\operatorname{time}(w)+1 \cap \operatorname{space}(u)=\operatorname{space}(w) \\
& \quad \cap(\operatorname{sym}(u), \operatorname{state}(u))= \\
& \quad \operatorname{next}(\operatorname{choice}(v), \operatorname{sym}(v), \operatorname{state}(v), \operatorname{sym}(w), \operatorname{state}(w), \operatorname{sym}(x), \operatorname{state}(x)))
\end{aligned}
$$

where the function next encodes the transition relation of $M$. The nondeterministic choice is determined by the value of the variable choice. Addition in this expression is modulo $N$. We are using the fact that the state and symbol at time $i+1$ and position $j$ depends only on the state and symbol at time $i$ and positions $j-1, j$, and $j+1$. The function next also encodes the fact that if the machine is not scanning tape cell $j$ at time $i$, then the symbol on tape cell $j$ is unchanged at time $i+1$.

By (8), any closed subhypergraph contains the start configuration of the computation. Inductively, assume any closed subhypergraph contains the first $i$ configurations. By (7), entries in configuration $i$ must agree on the value of the variable choice. Furthermore, given any $v, w$, and $x$ satisfying the premise of (9), there are exactly two $u$ satisfying the conclusion of (9) with different values of choice but otherwise identical. One of these must be in any closed subhypergraph.

We need to check that the accept state occurs someplace in the last row of the matrix. Since the machine has either accepted or rejected by time $N-1$, and

since we have already insured that the matrix accurately encodes a computation history, we need only check that the reject state $r$ does not occur in the last row. We use the formula

$$
\operatorname{time}(x)=N-1 \Rightarrow \operatorname{state}(x) \neq r .
$$

Finally, we define $C_{f}[u, v, w, x]$ to be the conjunction of (6)-(10).

We have argued that the problem of deciding whether a given system of constraints with one ternary and one nullary symbol is satisfiable is NEXPTIMEcomplete. It will follow from the result of the next section that the problem with one nullary and one binary symbol is also NEXPTIME-complete.

\subsection{Symbols of Greater Arity}

In this section we show that any system with symbols of arbitrary arity can be reduced to a system with a single binary symbol and a single constant. By the results of Section 6.4, it suffices to prove this result for a signature with one ternary symbol and one constant. This will establish the NEXPTIME-completeness of 
the satisfiability problem for systems with at least one constant and at least one symbol of arity two or greater.

Let $\Gamma=\{g, b\}$ and let $\Sigma=\{f, a\}$, where $a$ and $b$ are constants, $f$ is binary, and $g$ is ternary. Let $B, C_{b}$, and $C_{g}$ be formulas describing a hypergraph $H=$ $\left(U, E_{b}, E_{g}\right)$, where $E_{b}$ is unary and $E_{g}$ is quaternary. We will define a new hypergraph $\widehat{H}=\left(\widehat{U}, E_{a}, E_{f}\right)$ specified by formulas $\widehat{B}, C_{f}$, and $C_{a}$ such that $\widehat{H}$ has a closed induced subhypergraph iff $H$ does. The idea behind the construction is to encode one application of $E_{g}$ in $H$ with two nested applications of $E_{f}$ in $\hat{H}$.

The vertices of $\widehat{H}$ are $\widehat{U}=U \cup(U \times U)$. Elements of $U \times U$ are denoted $\langle u, v\rangle$. If $X$ is the set of variables used in the definition of $H$ such that elements of $U$ are truth assignments to $X$, then we can take $\hat{U}$ to be a set of truth assignments to $X \cup X^{\prime} \cup\{x\}$, where $X^{\prime}$ is a disjoint copy of $X$ and $x$ is a new variable whose sole purpose is to distinguish between $U$ and $U \times U$. We define $\widehat{B}, C_{f}$, and $C_{a}$ such that the following equations hold for any $s, t, u, v, w \in U$ and $p, q \in \widehat{U}$ :

$$
\begin{aligned}
\widehat{B}[p] & = \begin{cases}B[u], & \text { if } p=u, \\
B[u] \cap B[v], & \text { if } p=\langle u, v\rangle\end{cases} \\
C_{a}[p] & = \begin{cases}C_{b}[u], & \text { if } p=u, \\
0, & \text { if } p=\langle u, v\rangle\end{cases} \\
C_{f}[p, u, v] & = \begin{cases}1, \text { if } p=\langle u, v\rangle \\
0, \text { otherwise }\end{cases} \\
C_{f}[p,\langle u, v\rangle, w] & = \begin{cases}C_{g}[t, u, v, w], & \text { if } p=t, \\
0, & \text { if } p=\langle s, t\rangle\end{cases} \\
C_{f}[p, q,\langle u, v\rangle] & =1
\end{aligned}
$$

Here we are using notation similar to that defined at the end of Section 4.1, in which Boolean formulas are considered to be Boolean-valued functions on atoms or sequences of atoms.

To be more precise, the value of $x$ tells whether the truth assignment to $X \cup X^{\prime} \cup\{x\}$ encodes an element of $U$ (say if $x=0$ ) or an element of $U \times U$ (say if $x=1$ ). If the former, we only consider the truth assignment to $X$, which denotes an atom $u$. In that case we want $u$ in the hypergraph iff $u$ is an element of $U$; this is specified by the first alternative in (11). If the latter, then the truth assignment to $X$ denotes an atom $u$ and the truth assignment to $X^{\prime}$ denotes an atom $v$, and in that case we would like to have $\langle u, v\rangle$ in the hypergraph iff both $u$ and $v$ are elements of $U$; this is specified by the second alternative in (11).

Formally, (11) describes the following formula $\widehat{B}$ over $X \cup X^{\prime} \cup\{x\}$ :

$$
\widehat{B}=(\sim x \cap B) \cup\left(x \cap B \cap B\left(X^{\prime}\right)\right),
$$

where $X=\left\{x_{1}, \ldots, x_{n}\right\}, X^{\prime}=\left\{x_{1}^{\prime}, \ldots, x_{n}^{\prime}\right\}$, and $B\left(X^{\prime}\right)$ denotes $B$ with $x_{i}$ replaced by $x_{i}^{\prime}, 1 \leq i \leq n$.

Similarly, (12) describes the formula $C_{a}=\sim x \cap C_{b}$ over $X \cup X^{\prime} \cup\{x\}$. The remaining equations describe a formula $C_{f}$ over the variables

$$
X \cup X^{\prime} \cup\{x\} \cup Y \cup Y^{\prime} \cup\{y\} \cup Z \cup Z^{\prime} \cup\{z\} .
$$


Each of $p, q$ and $r$ in $C_{f}[p, q, r]$ is of the form either $u$ or $\langle u, v\rangle$, where $p$ is described by $X \cup X^{\prime} \cup\{x\}, q$ is described by $Y \cup Y^{\prime} \cup\{y\}$, and $r$ is described by $Z \cup Z^{\prime} \cup\{z\}$. The equations (13) and (14) say that $(u, v, w, x) \in E_{g}$ iff $(u,\langle v, w\rangle, x)$ and $(\langle v, w\rangle, v, w) \in E_{f}$.

The last equation (15) insures that for each $q \in \widehat{U}$ and $u, v \in U$, there is some $p \in \hat{U}$ for which $C_{f}[p, q,\langle u, v\rangle]$, so that closure in $\widehat{H}$ does not depend on $q$ and $\langle u, v\rangle$.

These formulas are easily derived from $B, C_{b}$, and $C_{g}$, and imply that the induced subhypergraph of $H$ on vertices $U^{\prime}$ is closed if and only if the induced subhypergraph of $\widehat{H}$ on vertices $U^{\prime} \cup\left(U^{\prime} \times U^{\prime}\right)$ is closed.

\section{Future Work}

We would like to extend these techniques to projection functions. For every symbol $f \in \Sigma_{n}$, one can define a family of projection functions $f^{-1}, \ldots, f^{-n}$ with semantics

$$
\sigma\left(f^{-i}(E)\right)=\left\{t_{i} \mid \exists t_{1}, \ldots, t_{i-1}, t_{i+1}, \ldots, t_{n} f t_{1} \ldots t_{n} \in \sigma(E)\right\}
$$

Algorithms for solving special cases of set constraints with projections are known $[18,14,12,6]$. Projection functions subsume negative constraints because the constraint system $\mathcal{S} \cup\left\{b \subseteq f^{-1}(f b y)\right\}$ is satisfiable only if $\mathcal{S}$ is satisfiable with $y \neq 0$. The results of $[1,11,19,9]$ on negative constraints are presumably a step towards solving systems with projections.

\section{Acknowledgements}

We thank the referees for their thorough reading and helpful comments.

Part of this research was done while the first and third authors were affiliated with the IBM Almaden Research Center, San Jose, California, and while the second author was on sabbatical at Aarhus University, Denmark. The support of the Danish Research Academy, the National Science Foundation under grant CCR-9317320, the John Simon Guggenheim Foundation, and the U.S. Army Research Office through the ACSyAM branch of the Mathematical Sciences Institute of Cornell University under contract DAAL03-91-C-0027 is gratefully acknowledged.

A previous version of this paper appeared as [5].

\section{References}

1. A. Aiken, D. Kozen, and E. Wimmers. Decidability of systems of set constraints with negative constraints. Technical Report 93-1362, Computer Science Department, Cornell University, June 1993. 
2. A. Aiken and B. Murphy. Implementing regular tree expressions. In Proc. 1991 Conf. Functional Programming Languages and Computer Architecture, pages 427447, August 1991.

3. A. Aiken and B. Murphy. Static type inference in a dynamically typed language. In Proc. 18th Symp. Principles of Programming Languages, pages 279-290. ACM, January 1991.

4. A. Aiken and E. Wimmers. Solving systems of set constraints. In Proc. 7th Symp. Logic in Computer Science, pages 329-340. IEEE, June 1992.

5. Alexander Aiken, Dexter Kozen, Moshe Vardi, and Edward Wimmers. The complexity of set constraints. Cornell University tech. report 93-1352, May 1993.

6. L. Bachmair, H. Ganzinger, and U. Waldmann. Set constraints are the monadic class. In Proc. 8th Symp. Logic in Computer Science, pages 75-83. IEEE, June 1993.

7. J. A. Brzozowski and E. Leiss. On equations for regular languages, finite automata, and sequential networks. Theor. Comput. Sci., 10:19-35, 1980.

8. A. Chandra, D. Kozen, and L. Stockmeyer. Alternation. J. Assoc. Comput. Mach., 28(1):114-133, 1981.

9. W. Charatonik and L. Pacholski. Negative set constraints with equality. In Proc. 9th Symp. Logic in Computer Science. IEEE, July 1994. To appear. Also, MaxPlanck-Institut für Informatik Technical Report MPI-I-93-265.

10. R. Gilleron, S. Tison, and M. Tommasi. Solving systems of set constraints using tree automata. In Proc. Symp. Theor. Aspects of Comput. Sci., volume 665, pages 505-514. Springer-Verlag Lect. Notes in Comput. Sci., February 1993.

11. R. Gilleron, S. Tison, and M. Tommasi. Solving systems of set constraints with negated subset relationships. In Proc. 34th Symp. Foundations of Comput. Sci, pages 372-380. IEEE, November 1993.

12. N. Heintze and J. Jaffar. A decision procedure for a class of set constraints. In Proc. 5th Symp. Logic in Computer Science, pages 42-51. IEEE, June 1990.

13. N. Heintze and J. Jaffar. A finite presentation theorem for approximating logic programs. In Proc. 17th Symp. Principles of Programming Languages, pages 197209. ACM, January 1990.

14. N. D. Jones and S. S. Muchnick. Flow analysis and optimization of LISP-like structures. In Proc. 6th Symp. Principles of Programming Languages, pages 244-256. ACM, January 1979.

15. K. Marriott and M. Odersky. Systems of negative Boolean constraints. Technical Report YALEU/DCS/RR-900, Computer Science Department, Yale University, April 1992.

16. P. Mishra. Towards a theory of types in PROLOG. In Proc. 1st Symp. Logic Programming, pages 289-298. IEEE, 1984.

17. P. Mishra and U. Reddy. Declaration-free type checking. In Proc. 12th Symp. Principles of Programming Languages, pages 7-21. ACM, 1985.

18. J. C. Reynolds. Automatic computation of data set definitions. In Information Processing 68, pages 456-461. North-Holland, 1969.

19. K. Stefánsson. Systems of set constraints with negative constraints are NEXPTIME-complete. In Proc. 9th Symp. Logic in Computer Science. IEEE, June 1994. To appear. Also Cornell University TR93-1380, August 1993.

20. J. Young and P. O’Keefe. Experience with a type evaluator. In D. Bjørner, A. P. Ershov, and N. D. Jones, editors, Partial Evaluation and Mixed Computation, pages 573-581. North-Holland, 1988. 
This article was processed using the $\mathrm{IAT}_{\mathrm{E}} \mathrm{X}$ macro package with LLNCS style 